\title{
Outcomes of Patients Treated with the Everolimus- versus the Paclitaxel-Eluting Stents in a Consecutive Cohort of Patients at a Tertiary Medical Center
}

\author{
Nicolas W. Shammas, MD, MS ${ }^{1}$ Gail A. Shammas, BS, RN ${ }^{1} \quad$ Elie Nader, MD ${ }^{1} \quad$ Michael Jerin, $\mathrm{PhD}^{1}$ \\ Luay Mrad, MD ${ }^{1}$ Nicholas Ehrecke ${ }^{1}$ Waheeb J. Shammas ${ }^{1}$ Cara M. Voelliger, BS ${ }^{1}$ Alexander Hafez ${ }^{1}$ \\ Ryan Kelly ${ }^{1}$ Emily Reynolds ${ }^{1}$ \\ ${ }^{1}$ Midwest Cardiovascular Research Foundation, Davenport, lowa \\ Int J Angiol 2013;22:165-170. \\ Address for correspondence Nicolas W. Shammas, MD, MS, FACC, \\ FSCAI, Midwest Cardiovascular Research Foundation, 1622 E Lombard \\ Street, Davenport, IA 52803 (e-mail: Shammas@mchsi.com).
}

\begin{abstract}
Keywords

- coronary stent

- drug-eluting stent

- paclitaxel

- everolimus

- outcome

- target lesion revascularization

- stent thrombosis

In this study, we compare the outcomes of the paclitaxel-eluting stent (PES) versus the everolimus-eluting stent (EES) treated patients at a tertiary medical center and up to 2 years follow-up. Unselected consecutive patients were retrospectively recruited following stenting with PES (159 patients) or EES (189 patients). The primary endpoint of the study was target lesion failure (TLF), defined as the combined endpoint of cardiac death, nonfatal myocardial infarction (MI), or target lesion revascularization (TLR). Secondary endpoints included target vessel revascularization (TVR), TLR, target vessel failure (TVF), acute stent thrombosis (ST), total death, cardiac death, and nonfatal MI. Patients treated with the PES stent had less congestive heart failure and restenotic lesions, but a higher prevalence of longer lesions, nonleft main bifurcations, required more stents per patient $(4.3 \pm 2.8$ vs. $2.9 \pm 2.1)$. TLF occurred in $32.3 \%$ PES versus $21.5 \% \operatorname{EES}(p=0.027)$. The secondary unadjusted endpoints for PES versus EES, respectively, were TVF 38.6 versus $30.7 \%(p=0.140)$, TVR 35.7 versus $26.5 \%$ $(p=0.079)$, definite and probable ST 1.2 versus $0.0 \%$, nonfatal MI 4.5 versus $4.2 \%$, and mortality 9.6 versus $4.0 \%$. Logistic regression analysis showed that the numbers of stents per patient $(p=0.001)$, age $(p=0.01)$, and renal failure $(p=0.045)$ were independent predictors of TLF. Using univariate analysis, EES had lower TLF than PES in a cohort of unselected patients undergoing percutaneous coronary intervention at 2 years follow-up. Multivariate analysis showed that the numbers of stents per patient, age, and renal failure, but not stent type, were predictors of TLF.
\end{abstract}

The paclitaxel-eluting stent (PES) Taxus Liberte (Boston Scientific, Natick, MA) and the everolimus-eluting stent (EES) Xience (or Promus) (Abbott Laboratories, Abbott Park, IL) are second generation drug-eluting stents (DES) that outperformed bare metal stents in reducing restenosis and target lesion revascularization (TLR). ${ }^{1-4}$

Randomized trials have shown that EES is superior to PES stents in reducing the composite endpoint of target lesion failure (TLF), stent thrombosis (ST), myocardial infarction (MI), and TLR. ${ }^{5-7}$ The diabetic subgroup, however, failed to show an advantage of EES over PES. ${ }^{5,8}$ Furthermore, in real life registries where patient selection was left to the interventionalist rather than limited by a set of inclusion and exclusion criteria of a randomized trial, EES was safe and effective but did not show an advantage over PES. ${ }^{9,10}$ published online

June 27, 2013
Copyright $(2013$ by Thieme Medical Publishers, Inc., 333 Seventh Avenue, New York, NY 10001, USA. Tel: +1(212) 584-4662.
DOI http://dx.doi.org/ 10.1055/s-0033-1347931. ISSN 1061-1711. 
Table 1 Demographics and clinical variables

\begin{tabular}{|c|c|c|c|c|c|}
\hline & $n^{\mathrm{a}}$ & Paclitaxel & $n^{\mathrm{a}}$ & Everolimus & $p$ value \\
\hline Age (years) & 158 & $67.2 \pm 12.2$ & 188 & $65.6 \pm 11.4$ & 0.213 \\
\hline Body mass index & 159 & $30.6 \pm 6.9$ & 186 & $30.9 \pm 6.6$ & 0.701 \\
\hline Male (\%) & $96 / 159$ & 60.4 & $125 / 189$ & 66.7 & 0.314 \\
\hline New York Heart Class (\%) & 158 & & 188 & & NA \\
\hline No symptoms/Class I & 145 & 91.8 & 157 & 83.5 & \\
\hline Class II & 7 & 4.4 & 26 & 13.8 & \\
\hline Class III & 4 & 2.5 & 3 & 1.6 & \\
\hline Class IV & 2 & 1.3 & 2 & 1.1 & \\
\hline New York Heart Class (symptomatic patients) & $16 / 158$ & 10.1 & $52 / 188$ & 27.7 & 0.001 \\
\hline Prior percutaneous coronary intervention (\%) & $103 / 159$ & 64.8 & $141 / 188$ & 75.0 & 0.045 \\
\hline Prior coronary artery bypass surgery (\%) & $32 / 159$ & 20.1 & $46 / 189$ & 24.3 & 0.369 \\
\hline Previous myocardial infarction (\%) & $53 / 159$ & 33.3 & $73 / 189$ & 38.6 & 0.316 \\
\hline Family history of premature CAD (\%) & $65 / 157$ & 41.4 & $75 / 185$ & 40.5 & 0.912 \\
\hline Renal failure (\%) & $19 / 159$ & 11.9 & $8 / 185$ & 4.3 & 0.014 \\
\hline Peripheral vascular disease (\%) & $25 / 159$ & 15.7 & $21 / 189$ & 11.1 & 0.266 \\
\hline History of hypertension (\%) & $133 / 159$ & 83.6 & $152 / 189$ & 80.4 & 0.486 \\
\hline Cerebrovascular disease (\%) & $4 / 158$ & 2.5 & $9 / 189$ & 4.8 & 0.396 \\
\hline Hyperlipidemia (\%) & $144 / 159$ & 90.6 & $160 / 189$ & 84.7 & 0.108 \\
\hline History of smoking (current and ex-smoker) (\%) & $99 / 159$ & 62.3 & $120 / 188$ & 63.8 & 0.823 \\
\hline Diabetes mellitus (\%) & $60 / 159$ & 37.7 & $69 / 188$ & 36.7 & 0.911 \\
\hline
\end{tabular}

Abbreviations: CAD, coronary artery disease; NA, nonapplicable.

${ }^{a}$ Denominator reflects number of patients with data available.

Long-term randomized studies also indicated that eventfree survival continues to diverge beyond the first 3 years in favor of EES with consistent improvement in its long-term safety and efficacy. ${ }^{11}$ Registry data, on the other hand, is mostly limited to 1 year follow-up. ${ }^{9,10}$

In this single center study, we compare Taxus Liberte PES to Xience (Promus) EES for late outcome at 2 years in an unselected consecutive group of patients at a single tertiary medical center.

\section{Methods}

Unselected consecutive patients were retrospectively recruited from a single center following stenting with PES or EES. Both de novo and restenotic lesions were included. Exclusion criteria included patients with bypass graft stenting or who received mixed stents. There were no prespecified institutional criteria to the choice of the DES which was left to individual operator. The first 100 consecutive patients in each cohort underwent syntax scoring by an independent investigator blinded to patients' outcomes trained in syntax scoring using the online tutorial on the SYNTAX score Web site (www.syntaxscore.com) and performing an additional 50 cases under close supervision by an experienced cardiologist.

- Tables 1 and $\mathbf{2}$ show the demographics, procedural, and clinical variables collected by reviewing medical records.
Angiographic variables are shown in - Table $\mathbf{3}$ and were obtained by an independent blinded review of the angiograms to patients' clinical variables and outcomes. Ejection fraction (EF) was obtained from left ventriculography during the index angiographic procedure.

Follow-up was at 2 years from the index procedure using medical records, phone calls, or both. Patients were initially mailed a brief letter describing the protocol, followed by a phone call to obtain verbal consent to be part of the study (using an institutional review board [IRB]-approved standardized script). All events reported by patients were verified by cross reference to medical records. Patients who expired had their death certificate retrieved when possible to evaluate the cause of their death.

The primary outcome of the study was TLF, defined as cardiac death, nonfatal MI related to target vessel, and TLR. Secondary outcomes included target vessel revascularization (TVR), target vessel failure (TVF) (defined as cardiac death, nonfatal MI, and TVR), acute ST as defined by the Academic Research Consortium, ${ }^{12}$ nonfatal MI, and cardiac death.

\section{Statistical Analysis}

Descriptive analysis was performed on all variables. $t$ testing was used for continuous variables and chi-square testing for dichotomous variables. Univariate analysis compared the demographic, clinical, angiographic, and outcome variables 
Table 2 Indications for angiography and coronary artery distribution

\begin{tabular}{|c|c|c|c|}
\hline & Paclitaxel & Everolimus & $p$ value \\
\hline Number of patients treated $(n)$ & 159 & 189 & \\
\hline Number of vessels treated $(n)^{\mathrm{a}}$ & 322 & 304 & \\
\hline Number of segments treated $(n)^{\mathbf{b}}$ & 602 & 476 & \\
\hline Indications for angiography (\%) & & & $1.000^{\mathrm{c}}$ \\
\hline Unstable angina/NSTEMI & 46.1 & 49.5 & \\
\hline STEMI & 4.5 & 2.4 & \\
\hline Abnormal perfusion test with symptoms & 12.9 & 15.4 & \\
\hline Abnormal perfusion test with no symptoms & 6.2 & 6.3 & \\
\hline Cardiomyopathy/congestive heart failure & 1.1 & 0.5 & \\
\hline Staged intervention & 24.7 & 24.0 & \\
\hline Arrhythmias & 1.7 & 0.5 & \\
\hline Recent decrease in ejection fraction & 0.6 & 1 & \\
\hline Progressive dyspnea & 2.2 & 0 & \\
\hline Syncope & 0 & 0.5 & \\
\hline Distribution of coronary artery disease (per number of vessels) (\%) & & & 0.574 \\
\hline RCA & 30.1 & 21.1 & \\
\hline LM & 6.6 & 9.9 & \\
\hline LAD & 33.9 & 35.9 & \\
\hline RI & 2.3 & 4.6 & \\
\hline LCX & 27.1 & 28.6 & \\
\hline
\end{tabular}

Abbreviations: LAD, left anterior descending artery; LCX, left circumflex; LM, left main; NSTEMI, non-ST elevation myocardial infarction; RCA, right coronary artery; RI, ramus intermedius; STEMI, ST elevation myocardial infarction.

${ }^{a}$ The following were considered vessels per patient and counted when treated: LAD, LCX, RI, RCA, and LM.

${ }^{\text {b} T h e ~ f o l l o w i n g ~ s e g m e n t s ~ w e r e ~ c o n s i d e r e d ~ p e r ~ v e s s e l ~ a n d ~ c o u n t e d ~ w h e n ~ t r e a t e d: ~ L A D-p r o x i m a l, ~ m i d, ~ d i s t a l, ~ d i a g o n a l ~} 1$ and 2; LCX-proximal, distal, obtuse marginal 1 and 2, posterolateral branch 1 and 2; RCA-proximal, mid, distal, acute marginal, posterior descending artery, posterolateral 1 and 2; disease in LM and RI was considered as one segment per vessel.

${ }^{c}$ Acute coronary syndrome (unstable angina, NSTEMI, STEMI) versus all others.

between the two groups. Survival analysis (Kaplan-Meier) was performed for TLF over the 2 years follow-up. Logistic regression analysis with backward elimination was performed modeling for age, gender, diabetes, New York Heart Class, prior percutaneous coronary intervention (PCI), renal insufficiency, percent of patients with nonleft main (LM) bifurcating disease, ostial lesions or restenotic lesions, length of disease treated per patient, number of stents use per patient, and stent type. SPSS (IBM, NY) software was used to conduct the analysis.

\section{Results}

A total of 182 PES and 207 EES unselected patients were initially screened to be recruited to the study. Patients were excluded if they refused verbal consent required by the IRB ( $n=17$ PES; $n=10$ EES) or lost to follow-up ( $n=5$ PES; $n=8$ EES). A total of 159 PES patients (322 vessels, 602 segments) and 189 EES patients (304 vessels, 476 segments) were included in the final analysis.

Descriptive analysis on all patients is shown in - Table 1. There was a high incidence of patients who had prior PCI and prior bypass surgery in both cohorts. Approximately twothirds of patients were current or prior smokers and $37 \%$ were diabetics.

The indication of the procedure was similar in both groups with about half the patients treated for an acute coronary syndrome. The distribution of disease was also similar between both cohorts with 7 to $10 \%$ of patients had their LM treated ( $\boldsymbol{- T a b l e ~} \mathbf{2}$ ).

Angiographic and procedural variables are shown in -Table 3. Longer disease was treated in the PES group leading to more stents placed per patient compared with the EES cohort ( 4.3 vs. $2.9, p=0.001$ ). On the contrary, more patients had restenotic lesions in the EES versus PES cohort ( 42.9 vs. $18.9 \%, p=0.001$ ). The SYNTAX scores of the first 100 consecutive patients, which reflect angiographic complexity, were statistically similar between PES and EES $(20.3 \pm 13.9$ vs. $20.4 \pm 13.8, p=0.97$ ). EF was also similar in both groups.

At 2 years, follow-up was completed for $82.3 \%$ of all patients enrolled (Promus 92.9\% and Taxus 71.5\%). The primary unadjusted outcome of TLF occurred in 32.3\% PES versus $21.5 \%$ EES $(p=0.027)$ (-Fig. 1, - Table 4$)$. The secondary unadjusted endpoints for PES versus EES, respectively, 
Table 3 Angiographic and procedural variables

\begin{tabular}{|c|c|c|c|}
\hline & Paclitaxel & Everolimus & $p$ value \\
\hline Per patient analysis & $n=159$ & $n=188$ & \\
\hline Ejection fraction (\%), mean \pm SD & $\begin{array}{l}49.0 \pm 14.7 \\
(n=101)\end{array}$ & $\begin{array}{l}51.5 \pm 14.3 \\
(n=152)\end{array}$ & 0.142 \\
\hline Stents use per patient $(n)$, mean \pm SD & $4.3 \pm 2.8$ & $2.9 \pm 2.1$ & 0.001 \\
\hline Length of disease treated per patient $(\mathrm{mm})$, mean \pm SD & $85.2 \pm 56.4$ & $52.2 \pm 43$ & 0.001 \\
\hline Patients treated with restenotic lesions (\%) & 18.9 & 42.9 & 0.001 \\
\hline Percentage of patients with non-LM bifurcating disease (\%) & 64.8 & 50.5 & 0.009 \\
\hline SYNTAX (first 100 consecutive patient in each group), mean \pm SD & $20.3 \pm 13.9$ & $20.4 \pm 13.8$ & 0.967 \\
\hline Per vessel analysis & $n=322$ & $n=304$ & \\
\hline Stents used per vessel $(n)$, mean \pm SD & $2.2 \pm 1.4$ & $1.8 \pm 1.2$ & 0.001 \\
\hline Length of disease treated per vessel $(\mathrm{mm})$, mean $\pm \mathrm{SD}$ & $42.5+33.8$ & $32.4 \pm 26.4$ & 0.001 \\
\hline Per segment analysis & $n=602$ & $n=476$ & \\
\hline Prelesion severity (\%), mean \pm SD & $84.0 \pm 12.2$ & $84.5 \pm 11.9$ & 0.467 \\
\hline Postlesion severity (\%) & 0.0 & 0.0 & 1 \\
\hline Diameter $(\mathrm{mm})$, mean $\pm \mathrm{SD}$ & $3.0 \pm 0.6$ & $3 \pm 0.5$ & 0.635 \\
\hline Stents used per segment $(n)$, mean \pm SD & $1.2 \pm 0.4$ & $1.2 \pm 0.4$ & 0.550 \\
\hline Length of disease treated per segment $(\mathrm{mm})$, mean \pm SD & $22.8 \pm 13.5$ & $20.7 \pm 12.6$ & 0.008 \\
\hline Segments treated with restenotic lesions (\%) & 9.3 & 34.1 & 0.001 \\
\hline Ostial segments treated (\%) & 5.3 & 8.2 & 0.063 \\
\hline
\end{tabular}

Abbreviation: LM, left main.

were TVF 38.6 versus $30.7 \%(p=0.140)$, TVR 35.7 versus $26.5 \%(p=0.079)$, definite and probable ST 1.2 versus $0.0 \%$, nonfatal MI 4.5 versus $4.2 \%$, and mortality 9.6 versus $4.0 \%$. To account for differences between the two cohorts, multivariate analysis was performed using logistic regression with backward elimination. This showed that the numbers of stents per patient $(p=0.001)$, age $(p=0.01)$, and renal failure

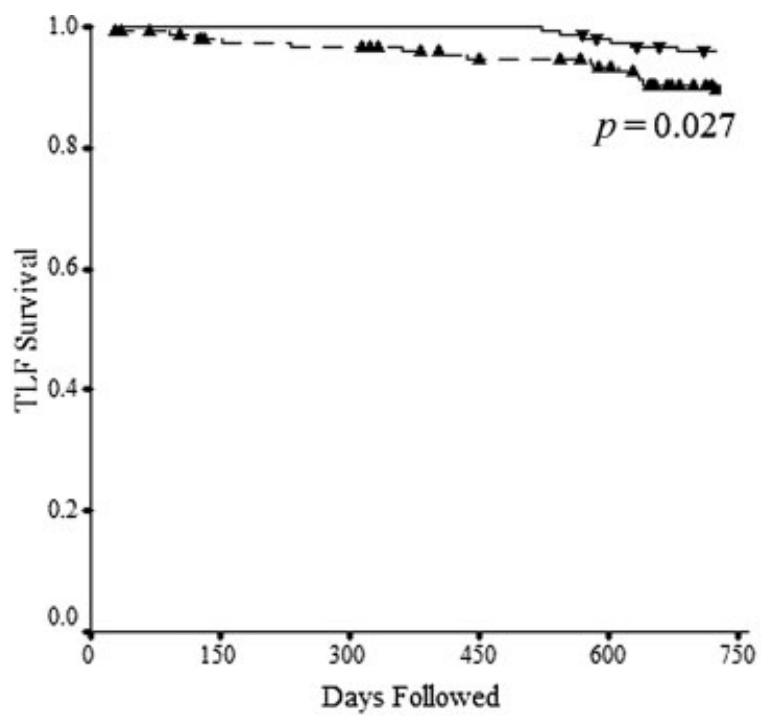

Fig. 1 Kaplan-Meier curve showing unadjusted TLF survival for the paclitaxel-eluting stent (interrupted line) versus the everolimus stent (full line). TLF, target lesion failure.
( $p=0.045)$ were independent predictors of TLF. Stent type was not predictive of TLF.

\section{Clinical History of Patients with Definite or Probable Acute Stent Thrombosis}

There were two patients who had definite or probable ST in the PES cohort. The first patient was a 60 -year-old man with a history of prior PCI and bypass surgery, hypertension, hyperlipidemia, current smoker, and diabetic. His index procedure was for an acute coronary syndrome involving the left circumflex artery with a $100 \%$ occlusion of a restenotic lesion. ST occurred 36 days postindex procedure. He was on clopidogrel and aspirin. His lesion was retreated successfully. The second patient was an 80-year-old woman with a history of prior $\mathrm{PCI}$, hypertension, hyperlipidemia, and diabetes mellitus. She had an EF of $20 \%$. Her index procedure was for an acute coronary syndrome involving a de novo LAD lesion. She had sudden cardiac death at day 28 after her procedure. She was on clopidogrel and aspirin.

\section{Discussion}

In this study, the primary unadjusted outcome of TLF occurred in $21.5 \%$ of EES versus $32.3 \%$ of PES ( $p=0.027$ ). Although this higher rate of TLF could be explained by the longer lesions treated and higher prevalence of non-LM bifurcation lesions in the PES group, the EES group also had a higher rate of restenotic lesions, a predictor of restenosis. Multivariate analysis does not show superior results of EES 
Table 4 Outcome of patients censored at 2 years follow-up (nonpropensity-matched analysis)

\begin{tabular}{|l|l|l|l|}
\hline & Paclitaxel & Everolimus & $p$ value \\
\hline TLR per segment (\%) & 17.7 & 9.5 & 0.001 \\
\hline TLR per vessel (\%) & 22.5 & 12.6 & 0.001 \\
\hline TLR per patient (\%) & 29.6 & 17.2 & 0.007 \\
\hline TVR per segment (\%) & 15.5 & 11.9 & 0.104 \\
\hline TVR per vessel (\%) & 28.2 & 18.3 & 0.004 \\
\hline TVR per patient (\%) & 35.7 & 26.5 & 0.079 \\
\hline Target lesion failure (\%) & 32.3 & 21.5 & 0.027 \\
\hline TLR & 29.1 & 17.2 & \\
\hline Cardiac death & 1.9 & 3.7 & \\
\hline Nonfatal MI & 4.5 & 4.3 & \\
\hline Target vessel failure (\%) & 38.6 & 30.7 & 0.140 \\
\hline Stent thrombosis (\%) & & & AA \\
\hline Definite or probable & 1.2 & 0.0 & \\
\hline Possible & 2.6 & 1.1 & 0.024 \\
\hline Percent of patients on clopidogrel on follow-up (\%) & 89.5 & 80 & 0.118 \\
\hline Percent of patients on aspirin on follow-up (\%) & 94.8 & 97.7 & \\
\hline Death classification on follow-up (\%) & & & NA \\
\hline No & 90.4 & 96.0 & \\
\hline Noncardiac & 3.8 & 2.0 & \\
\hline Unknown & 0.0 & 0.7 & 0.462 \\
\hline Cardiac sudden death probable & 2.0 & 0.6 & \\
\hline Cardiac nonstent thrombosis & 3.8 & 0.7 & \\
\hline Acute NSTEMl, nonfatal (\%) & 3.2 & 2.6 & \\
\hline Acute STEMl, nonfatal (\%) & 1.3 & & \\
\hline
\end{tabular}

Abbreviations: MI, myocardial infarction; NA, nonapplicable; NSTEMI, non-ST elevation myocardial infarction; STEMI, ST elevation myocardial infarction; TLR, target lesion revascularization; TVR, target vessel revascularization.

over PES at 2 years follow-up. This finding is in contrast with data from prospective randomized trials. In the SPIRIT III trial and at 2 years follow-up, patients treated with EES compared with PES had a significant $32 \%$ reduction in TVF (10.7 vs. $15.4 \% ; p=0.04$ ) and a $45 \%$ reduction in major adverse cardiac events (cardiac death, MI, or TLR; 7.3 vs. $12.8 \% ; p=0.004$ ). ${ }^{13}$ This benefit continued at 3 years. ${ }^{11}$ In SPIRIT IV, ${ }^{5}$ TLF was also reduced with EES compared with PES (4.2 vs. 6.8\%; $p=0.001$ ), a consistent finding in all subgroups except the diabetics (6.4 vs. $6.9 \% ; p=0.80$ ). Furthermore, in COMPARE, ${ }^{6}$ the primary composite endpoint of all-cause mortality, MI, and TVR at 1 year occurred in 6\% in EES versus $9 \%$ in PES $(p=0.02)$. Finally, a meta-analysis of randomized trials between EES versus PES ${ }^{7}$ also showed that EES had less MI, ST, and TVR than PES.

Our findings, however, are consistent with published realworld registries. ${ }^{9,10}$ When broader lesion categories are included, the difference between the two stents at 1 year follow-up becomes less apparent. In the REWARDS TLX multicenter registry, ${ }^{9}$ the primary composite endpoint of all-cause death, Q-wave MI, TVR, and ST at 1 year was similar between EES and PES (7.8 vs. $10.8 \% ; p=0.082$ ) with an overall trend toward more ST in the PES group (0.3 vs. $1.2 \% ; p=0.107)$. In the Washington Hospital Center experience of 6,615 patients treated with PES, EES, and sirolimuseluting stents, ${ }^{10}$ multivariate analysis showed so significant difference between EES versus PES in the rate of death and TVR. Definite ST was slightly higher with PES ( 0.0 vs. $0.7 \%$; $p=0.09$ ). Similarly, in our study, multivariate analysis showed stent type is not a predictor of TLF at 2 years. This finding could be attributed to the high prevalence of diabetics (37-38\%) in the two cohorts that may have reduced the advantage of EES over PES. ${ }^{5,8}$ Also, our study may have not been powered to detect statistical differences between PES versus EES at 2 years. Notably, TLF was higher in our study than reported for both PES and EES. This could be explained by the higher complexity of disease such as a high prevalence of diabetics, LM, long lesions, restenotic lesions, and bifurcations. Also, ST appears to be higher with PES than EES (1.3 vs. $0 \%$ ) consistent with both randomized trials and registry data.

\section{Limitations of the Study}

The study is limited by its retrospective nature and inherently selection bias. Also, the relatively small number of patients 
yielded significant baseline differences between the two groups. Although multivariate analysis was performed to model for independent predictors of TLF and adjust for differences between the EES versus PES cohorts, this cannot completely mitigate potential influence of these variables on the final outcome. In univariate analysis, EES outperformed PES which could not be seen in the multivariate model, a potentially false-negative finding given the small number of patients and baseline differences. Also, syntax scoring was limited to the first consecutive 100 patients in each cohort. However, the strikingly similar results between the two cohorts likely predict no difference in syntax scoring for the entire cohort. Furthermore, the follow-up of patients in the study was limited to 2 years, a relatively short period of time as differences between the two stents may not become statistically apparent until later follow-up. Finally, higher rates of TLR and TLF were seen compared with published data. This may be explained by a complex patient population with a large number of restenotic lesions, longer lesions, and high prevalence of LM and bifurcating disease.

\section{Acknowledgments}

The Midwest Cardiovascular Research Foundation (MCRF) has received research and educational grants from Medtronic, Abbott, and Boston Scientific. This study was supported in part by the Nicolas and Gail Research Fund at MCRF.

\section{References}

1 Grube E, Silber S, Hauptmann KE, et al. TAXUS I: six- and twelvemonth results from a randomized, double-blind trial on a slowrelease paclitaxel-eluting stent for de novo coronary lesions. Circulation 2003;107(1):38-42

2 Colombo A, Drzewiecki J, Banning A, et al. TAXUS II Study Group Randomized study to assess the effectiveness of slow- and moderate-release polymer-based paclitaxel-eluting stent for coronary artery lesions. Circulation 2003;108(7):788-794
3 Stone GW, Ellis SG, Cox DA, et al; TAXUS-IV Investigators. A polymer-based, paclitaxel-eluting stent in patients with coronary artery disease. N Engl J Med 2004;350(3):221-231

4 Stone GW, Ellis SG, Cox DA, et al; TAXUS-IV Investigators. One-year clinical results with the slow-release, polymer-based, paclitaxeleluting TAXUS stent: the TAXUS-IV trial. Circulation 2004; 109(16):1942-1947

5 Stone GW, Rizvi A, Newman W, et al; SPIRIT IV Investigators. Everolimus-eluting versus paclitaxel-eluting stents in coronary artery disease. N Engl J Med 2010;362(18):1663-1674

6 Kedhi E, Joesoef KS, McFadden E, et al. Second-generation everolimus-eluting and paclitaxel-eluting stents in real-life practice (COMPARE): a randomised trial. Lancet 2010;375 (9710):201-209

7 Alazzoni A, Al-Saleh A, Jolly SS. Everolimus-eluting versus paclitaxel-eluting stents in percutaneous coronary intervention: metaanalysis of randomized trials. Thrombosis 2012;2012:126369

8 Stone GW, Kedhi E, Kereiakes DJ, et al. Differential clinical responses to everolimus-eluting and Paclitaxel-eluting coronary stents in patients with and without diabetes mellitus. Circulation 2011;124(8):893-900

9 Waksman R, Ghali M, Goodroe R, et al. Percutaneous coronary intervention with second-generation paclitaxel-eluting stents versus everolimus-eluting stents in United States contemporary practice (REWARDS TLX Trial). Am J Cardiol 2012;110(8): 1119-1124

10 Mahmoudi M, Delhaye C, Wakabayashi K, et al. Outcomes after unrestricted use of everolimus-eluting stent compared to paclitaxel- and sirolimus-eluting stents. Am J Cardiol 2011;107(12): 1757-1762

11 Applegate RJ, Yaqub M, Hermiller JB, et al. Long-term (three-year) safety and efficacy of everolimus-eluting stents compared to paclitaxel-eluting stents (from the SPIRIT III Trial). Am J Cardiol 2011;107(6):833-840

12 Food and Drug Administration. Circulatory System Devices Panel Meeting. Available at: http://www.fda.gov/ohrms/dockets/ac/ cdrh06.html\#circulatory. Accessed February 9, 2007

13 Stone GW, Midei M, Newman W, et al; SPIRIT III Investigators. Randomized comparison of everolimus-eluting and paclitaxeleluting stents: two-year clinical follow-up from the Clinical Evaluation of the Xience $\mathrm{V}$ Everolimus Eluting Coronary Stent System in the Treatment of Patients with de novo Native Coronary Artery Lesions (SPIRIT) III trial. Circulation 2009;119(5):680-686 